\title{
Current in vitro approaches to assess nanoparticle interactions with lung cells
}

The respiratory tract is in constant contact with inhaled antigens from the external environment. In order to shape its line of defense, it is populated by various types of immune cells. Taking into account the scientific breakthroughs of nanomedicine and nanoparticle drug delivery, we can think of the respiratory tract as an ideal target organ to study and develop nanocarrier-based vaccines to treat respiratory tract disorders. Nanoparticles have been proven capable of specific cell targeting and, when suitably engineered, are able to induce an immunomodulatory effect. The aim of this review is to highlight in vitro approaches to the study of nanoparticle-lung immune cell interactions and recent advances in the targeting of immune cells using nanoparticle-based systems.

First draft submitted: 12 May 2016; Accepted for publication: 25 July 2016; Published online: 16 August 2016

Keywords: biomedical nanoparticles $\bullet$ immune modulation $\bullet$ in vitro approaches - lung immune cells $\bullet$ specific targeting

The rapid expansion of nanotechnology has resulted in the production of an impressive variety of nanoparticles (NPs) that have been defined by the European Network on the Health and Environmental Impact of Nanomaterials, according to ISO guidelines (ISO TC 229/TS 27687:2008), as objects with all three spatial dimensions on the nanoscale $(1-100 \mathrm{~nm})$. Due to their small size they possess high surface-to-volume ratios compared with bulk materials of the same chemical composition and their unique physicochemical properties allow implementation of NPs in a variety of biomedical applications, for instance as diagnostic or therapeutic agents, and in novel vaccine formulations [1,2]. Even though NPs intended for biomedical applications are part of the high risk, high payoff global nanotechnology phenomenon, the process of converting basic nanomedicine research into commercially viable products is likely to be long and difficult. Acceptance and integration of the products of nanotech- nology, particularly in nanomedicine, will be a challenge for a number of reasons. Not only approval by the regulatory agencies is required but also manufacturing processes require significant technological and cultural adjustments on the part of the producers [3].

Potential biomedical application of NPs can occur by means of the lungs via inhalation, the gastrointestinal tract via digestion, the skin via direct application or the blood vessels via intravenous administration [4-6]. Although most of the recent developments in the field of biomedical NPs has considered intravenous injection as the major administration path, simultaneously, the lungs are considered a promising pathway for delivering NPs as biomedical therapeutic or diagnostic agents via inhalation [7] due to their vast epithelial surface area of $>150 \mathrm{~m}^{2}$ and an extremely thin air-blood barrier of less than $1 \mu \mathrm{m}[7,8]$. The respiratory tract is functionally subdivided into a conducting region consisting of the airways (trachea, bronchi and
Kleanthis Fytianos ${ }^{\ddagger}, 1$, Barbara Drasler ${ }^{\ddagger 1}$, Fabian Blank ${ }^{2}$, Christophe von Garnier², Emilie Seydoux², Laura Rodriguez-Lorenzo', Alke Petri-Fink ${ }^{1} \&$ Barbara Rothen-Rutishauser $*, 1$ 'Adolphe Merkle Institute, University of Fribourg, Fribourg, Switzerland ${ }^{2}$ Respiratory Medicine, Inselspital, and Department of Clinical Research, University of Bern, Bern, Switzerland *Author for correspondence: Tel.: +41263009502 barbara.rothen@unifr.ch ${ }^{\ddagger}$ Authors contributed equally

$\begin{array}{ll}\text { Future } & \text { fSg } \\ \text { Medicine } & \text { part of }\end{array}$ 
bronchioles), and the actual respiratory part where gas exchange occurs (alveoli) [9] (Figure 1). Following inhalation, airborne particles deposit in the different regions of the respiratory tract in a size-dependent manner [10,11]. Larger particles $(1-10 \mu \mathrm{m})$ preferentially deposit in trachea and bronchi, whereas smaller particles (i.e., NPs) tend to deposit in the peripheral lung regions (alveoli). Therefore, depending on the size of the particles, different compartments of the respiratory tract may be targeted. Once deposited, particles interact with pulmonary surfactants and are displaced by wetting forces into the aqueous hypophase [12,13], where the interaction with pulmonary cells occurs. Then, particles can be cleared either via phagocytosis by macrophages or via mucociliary clearance within the conducting airways or translocation through the airblood tissue barrier in the more peripheral regions [14]. The latter might result in NPs entering systemic circulation $[15,16]$ and distribution of particles to multiple organs [17]. Depending on the particle properties and the rate of clearance, interaction with lung cells may cause cytokine-induced inflammation [18].

To maintain its homeostasis and thus to protect the lungs and subsequently the whole organism from invading pathogens, the lung is home to a variety of immune cell populations, such as phagocytic cells and antigen presenting cells, that is, macrophages and dendritic cells (DCs) [19,20]. The primary role of immature DCs, strategically positioned within the mucosal tissue, is to sample antigen by extending protrusions into the airway or alveolar lumen [21]. In this process, depending on the nature of the antigen, DCs may get activated via pattern recognition receptors and enter maturation process [22]. In case of activation/maturation DCs rapidly migrate to the draining lymph nodes [21-23]. In the course of maturation antigens are processed by the DCs and presented to naive T cells via the major histocompatibility complex (MHC II) in combination with upregulated co-stimulatory molecules on the DCs surface (CD40, CD80, CD86) and the release of cytokines. The type and combination of the cytokines released will determine the nature of effector $\mathrm{T}$ cells induced (Th1, Th2, Treg and Th17) [22] (Figure 2).

Therefore, specific targeting of lung-resident cells by NPs has been of great interest over the past decade [24]. It has been reported that adequately engineered NPs can specifically target cell populations in the different compartments, that is, conducting area (or lung parenchyma) of the respiratory tract [25], or via receptor-mediated mechanisms; the latter being the most common mechanisms for this purpose [26]. When NPs encounter cells, they can trigger immunomodulatory effects $[25,26]$. The latter effect is desirable in the case of NP-based drug carriers/vaccines where NPs may serve as adjuvants (as reviewed elsewhere [26-29]). On the other hand, NPs might also be accidentally introduced into therapeutics, thus significantly contributing to therapeutic efficiency [30]. Nevertheless, NP immunogenicity as well as antigenicity (i.e., the ability to induce immune response, and the ability to be specifically recognized by the antibodies, respectively) has recently been reviewed in detail by Ilinskaya and Dobrovolskaia [31], concluding that by tuning the physicochemical properties of NP-based carriers it is possible to either stimulate the immune system or to avoid its recognition. On the other hand, from the biotechnological perspective, improving our understanding of the undesirable immunogenicity and antigenicity of nanoparticulate-contaminated therapeutics is an essential future step required for the safe design of the nanomedicine products [31]. Moreover, the adverse side effects of NPs encountering immune cells also need to be considered, such as immune-mediated destruction or rejection, which can result in the elimination of NPs and/or immunotoxicity, and thus possible damage to the immune system and pathological states: a phenomenon that has been reviewed in the past [32]. Herein we discuss the basic functions of the lung-resident immune cells, such as macrophages and DCs, but also consider cells which can be activated by down-stream reactions in the draining lymph nodes, such as B or $\mathrm{T}$ cells as well as the in vitro approaches used in the fields of NP-based drug delivery, immune-modulation and specific cell targeting.

The aim of the review is to show the state-of-theart of the in vitro human lung immune cell-model systems, applicable for a first evaluation of biomedical NPs aimed to be used for therapeutic and diagnostic purposes as well as to provide an overview of the current knowledge of specifically designed NPs intended for modulation of different lung diseases. The interactions between NPs and various components of the immune system have become an active area of research in nanotechnology (recently reviewed in Dobrovolskaia et al. [30]). However, there is a lack of systematic reviews available on the routes of administration of biomedical NPs in human lungs, and their biocompatibility with pulmonary immune cells per se. The majority of the literature data on NP-based pulmonary treatments has been derived from mouse (or other mammal) studies, but there is currently incomplete data on the interaction between NPs and the human pulmonary immune system. However, for a better appraisal of the subject, we have occasionally incorporated findings and interpretations arising from studies on other mammalian objects. Finally, taking together the anatomy and physiology of the respiratory tract, the unique functions of the different lung cell 


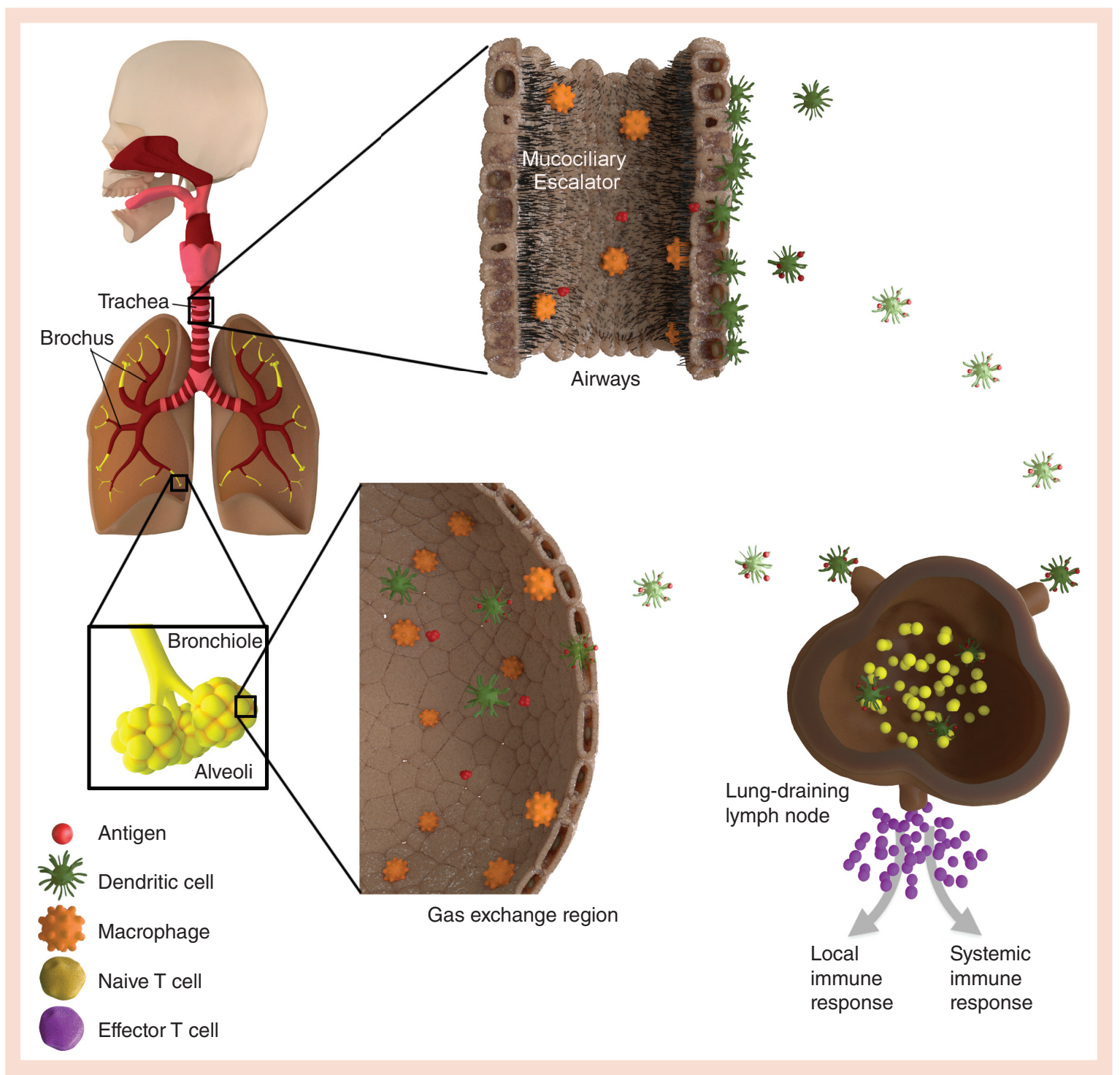

Figure 1. Schematic presentation of the structural organisation of the human respiratory immune system. The upper respiratory epithelium, lining the inner surface of the trachea, bronchi and bronchioles, is composed of a pseudostratified layer of ciliated cells, mucus-producing cells and secretory cells, and is responsible for the elimination of potential pathogens and foreign particles with the action of the mucociliary escalator. The distal regions of the lung epithelium, the alveolar septa, represent the site of the gas exchange. In both regions, macrophages are located at the apical side of the epithelial layer and protect it from the inhaled antigen cells by phagocytosis. Dendritic cells will capture antigens, process and present antigen peptide to naive T cells, and trigger their differentiation into antigen-specific effector T cells.

populations and the advancements made in preparing sophisticated NP systems, we propose that there is plenty of room for the development of novel pulmonary drug delivery approaches for the treatment and diagnosis of pulmonary disorders.

\section{General principles of NP systems for medical applications}

Intense research efforts have been focused on the development of NPs for novel biomedical applications.
However, prior to considering complex in vivo studies or clinical trials with such nanocarriers, a comprehensive in-depth knowledge of NP-cell interactions on the single-cell level is required. For this, an exact control and thorough characterization of both the materials and the cell-model systems must be employed.

Regarding the synthesis and characterization of medically relevant NPs, research has mainly been focused on revealing any physicochemical related cell responses as well as on the potential adverse effects of 


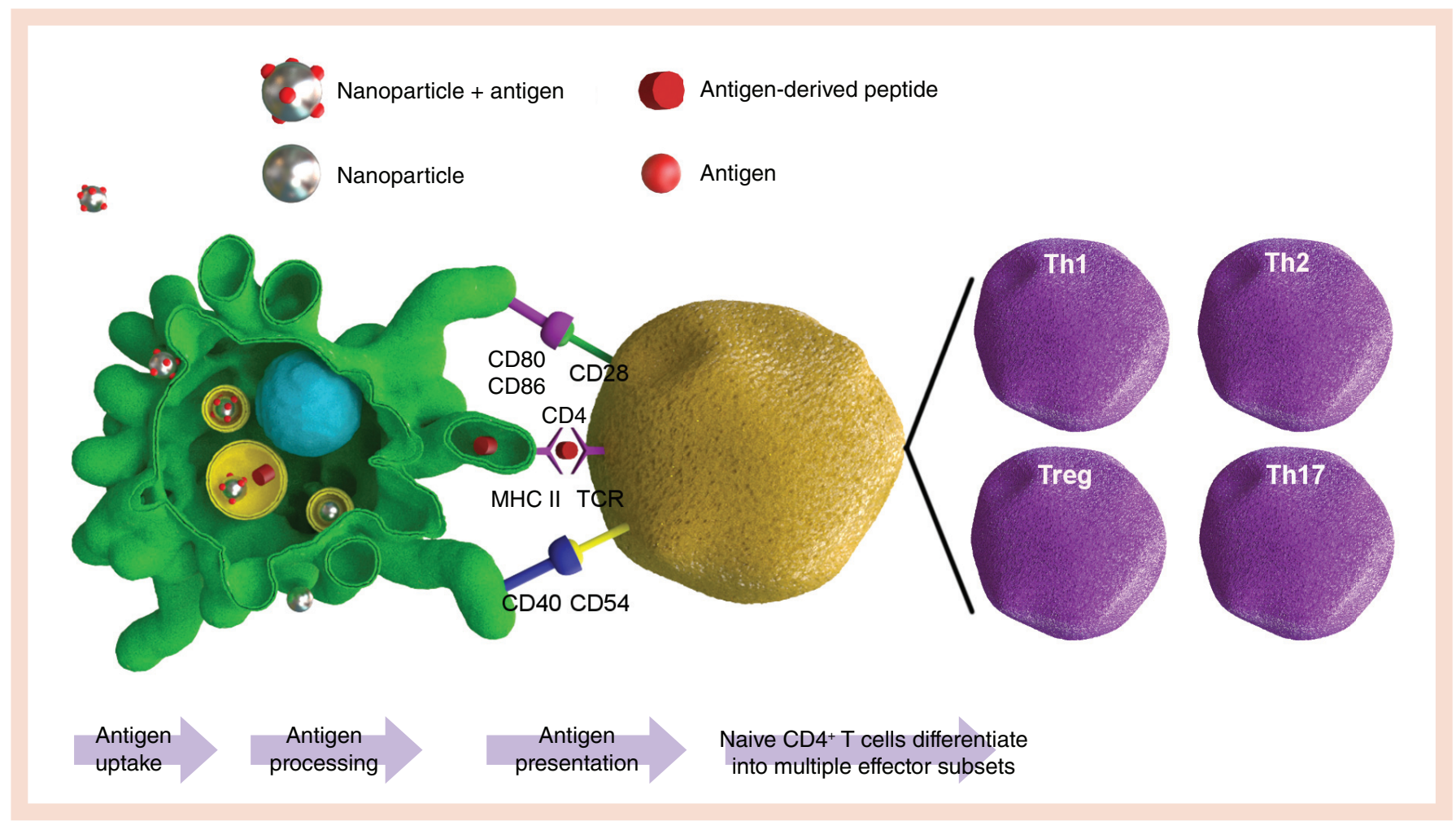

Figure 2. Antigen processing and presentation by dendritic cells to the naive T cells. Upon the antigen (or the nanoparticle-antigen complex) uptake, the antigen-derived peptide may be presented to the naive T cells via the major histocompatibility complex (MHC II) in combination with co-stimulatory molecules (CD40, CD80, CD86) and cytokines, released by T-helper cells. The activated T cells differentiate into different subsets of effector T cells (Th1, Th2, Treg and Th17).

CD80/86/28/40/54: Clusters of differentiation, i.e., proteins involved as a co-stimulatory signals in T-cell activation; MHC II: Major histocompatibility complex class II molecules are heterodimeric proteins expressed in antigen presenting cells and involved in the i.e., MHC class II-dependent pathway of antigen presentation (phagocyted compounds); Th1, Th2, Treg and Th17: Different subsets of T-helper cells, which are responsible for production of cytokines, involved in activation of T cells (among other processes).

those NPs in biological systems [33]. Both for basic and applied scientific research it is particularly important to address the fundamental question regarding the state of the material in a complex biological environment, such as cell culture media containing, for example, proteins, lipids or electrolytes, which might essentially alter the physicochemical properties of the NPs $[34,35]$ and consequently impact their cellular interaction in vitro and in vivo. Moreover, the interactions of NPs with cells depends also on their physicochemical properties, such as size, shape and surface charge [36,37]. In addition, due to the high complexity of the cellular systems, each individual cell type might react differently to another when encountering the same NPs [25].

An example of NPs with potential biomedical applications are gold NPs, which are chemically stable, have high electron density and possess high affinity toward biomolecules, such as amino acids, proteins and DNA, rendering those particles suitable candidates for drug carriers and imaging reagents [38]. Promising in vitro results have been obtained with conjugated gold NPs in cancer research for detection and treat- ment of tumor cells in vitro [39,40]. Moreover, diverse biodegradable and biocompatible polymer-based NPs, such as polylactic-co-glycolic acid (PLGA), have been shown to improve vaccination efficiency and provide heightened potency, thus present promising antigen delivery vehicles for diverse vaccine applications [41-43]. Another example of promising biomedical NPs are the super-paramagnetic iron oxide NPs (SPIONs) which have routinely been utilized as contrast agents in magnetic resonance imaging, in particular for transverse (or spin-spin) relaxation time (T2) imaging [44].

\section{Inhalative nanocarriers: state-of-the-art}

General knowledge about immune responses upon exposure to NPs (i.e., immunostimulation and immunosuppression) is very limited, particularly with respect to lung-resident immune cells. Since upon inhalation NPs can be recognized by the organism as foreign materials, surface modification of NPs is essentially required in order to avoid or minimize any potential undesired pathological conditions (i.e., cell activation and hypersensitivity). 
There has been a growing interest in the field of NPbased drug delivery research in the respiratory system, which is presented in Table 1. Various types of nanomaterials (i.e., lipid-based, polymers, metals coated with polymer, antibody-conjugated, carbon) have been used as agents in various pulmonary vaccines, where the DCs are predominantly the main target, as well as for targeting alveolar macrophages, since pathogens such as bacteria or fungi utilize them as a breeding ground (for a review see [45]).

In order to fully understand the interactions between NPs and lung-resident immune cells, careful design thorough characterization and proper control of all the synthetic steps is essential in the manufacture of NPs suitable for biomedical applications [56]. In addition to that, the potential effects of NPs on the cells must be evaluated, which requires a detailed cellular characterization; for this reason, well-studied in vitro model sys- tems have to be applied in order to achieve realistic and accurate data [57].

\section{The human lung immune system}

The immune system is intended to protect the organism from possibly harmful materials that are recognized as foreign substances (antigens) [58]. First, the innate immune system is characterized as the organism's 'first line of defense' and it thus provides rapid sensing and elimination of pathogens. It consists of the physical barriers (epithelial, mucus and surfactant layers) which cover the respiratory tract as well as the cytokines (which modulate cellular functions) and the membrane receptors (to which the antigens can bind), which are both primarily considered as parts of the innate immune system [59]. Second, the adaptive immune system provides an antigen-specific defense, and is responsible for generation of the immunological memory. Its

Table 1. A brief summary of various types of nanocarrier-based methods that have been investigated for inhalationoriented drug and vaccine delivery in the lungs.

\begin{tabular}{|c|c|c|c|c|}
\hline NPs type & Cell targeted & Immunological function & Study (year) & Ref. \\
\hline Lipid and polymer NPs & APCs of the lymphoid system & $\begin{array}{l}\text { - Enhancement of antigen } \\
\text { immunoavailability } \\
\text { - Antigen signal recognition } \\
\text { - Immunomodulation }\end{array}$ & Csaba et al. (2009) & [47] \\
\hline PEGylated dendrimers & Lung tissue/blood & Specific site/drug delivery & Ryan et al. (2013) & [48] \\
\hline PLGA & $\begin{array}{l}\text { Diphtheria infected cells in } \\
\text { the upper lung compartment }\end{array}$ & Enhanced IgA production & Muttil et al. (2010) & [49] \\
\hline PLA and PLGA & $\begin{array}{l}\text { Cell targeting via Hepatitis B } \\
\text { antigen antibody }\end{array}$ & $\begin{array}{l}\text { Enhancement of immune } \\
\text { responses }\end{array}$ & Thomas et al. (2010) & [50] \\
\hline Silica NPs & Lung tumor cells & Anticancer & Taratula et al. (2011) & [51] \\
\hline $\begin{array}{l}\text { Mannose-functionalized } \\
\text { polymer NPs }\end{array}$ & Bone marrow mouse DCs & Immune stimulation & $\begin{array}{l}\text { Carrillo-Conde et al. } \\
\text { (2011) }\end{array}$ & [52] \\
\hline $\begin{array}{l}\text { Gold cores coated with } \\
\text { galactofuranose conjugated } \\
\text { with anti-DC-SIGN antibodies }\end{array}$ & $\begin{array}{l}\text { Human blood monocyte } \\
\text { derived dendritic cells }\end{array}$ & $\begin{array}{l}\text { - Activation, maturation } \\
\text { - Release of IL- } 6 \text { and TNF- } \alpha\end{array}$ & Chiodo et al. (2014) & [54] \\
\hline \multirow[t]{3}{*}{ Polystyrene particles } & \multirow[t]{3}{*}{$\begin{array}{l}\text { Mouse, respiratory tract } \\
\text { dendritic cells }\end{array}$} & $\begin{array}{l}\text { - Enhanced uptake of smaller } \\
\text { NPs }\end{array}$ & Blank et al. (2013) & [25] \\
\hline & & $\begin{array}{l}\text { - Enhanced activation of } \\
\text { antigen (OVA) specific T cells }\end{array}$ & Seydoux et al. (2014) & [55] \\
\hline & & $\begin{array}{l}\text { - Reduced inflammatory } \\
\text { symptoms when applied prior } \\
\text { to sensitization and challenge }\end{array}$ & Hardy et al. (2012) & [19] \\
\hline
\end{tabular}


main feature is a tightly regulated interplay between the antigen presenting cells, such as macrophages or DCs, and the main actors of the adaptive immunity, $\mathrm{T}$ and $\mathrm{B}$ lymphocytes; the latter are responsible for the antigen-specific receptor-mediated antibody production. Generally, the adaptive immune system acts after the innate immune response when B and T-cell receptors become specific for the antigen after their first encounter and start to clonally expand. A proportion of these cells may persist as memory B or T cells, and in the case of re-exposure to the same pathogen, these receptors provide a capability of rapid response (based on the principle of the immunological memory) [60].

Upon entering the respiratory tract, the fate of deposited NPs not only depends on their physicochemical properties (e.g., particle size) [61] but also on the exposed cell type, that is, phagocytes (macrophages), antigen-presenting cells and epithelial or endothelial cells. Inhaled particles in the airways may trigger an early response of the innate immune system (epithelium, surfactant proteins, phagocytes such as neutrophils and macrophages, natural killer cells, mast cells, eosinophils, basophils and innate lymphoid cells), followed by a downstream response of the adaptive immune system's secretion of antibodies and T-cell responses) [46].

\section{Cells of the lung immune system \& selected cell models}

An overview of the important structural organization of the lung immune system is presented in Figures $1 \& 2$. The theory behind the basic principles of immunology is beyond the scope of this review, but has been elegantly presented elsewhere $[59,60]$. However, a brief introduction of the individual components of the immune system in lungs is given below.

\section{Macrophages}

Macrophages are professional phagocyte cells of the immune system (i.e., innate immunity) and their main function is to engulf antigens. They are involved in wound healing (i.e., tissue repair) and immune regulation (i.e., cell signaling and cytokine secretion) [62]. Airway as well as alveolar macrophages is located at the apical side of the epithelial layer in the air-blood interface and protect it from inhaled antigens (i.e., toxic and allergic particles) by macrophage-mediated clearance (i.e., phagocytosis) [63].

For the purpose of in vitro experiments to test their interaction with NPs, monocytes can be isolated from human buffy coat and differentiated into mature macrophages (monocyte-derived macrophages) with the addition of monocyte colony stimulating factor [64]; production of such primary cells can be very reproducible and represent a reliable in vitro system. Regarding the cell lines, the most commonly used are J774.1a (mouse macrophages derived from ascites) and THP-1 (human peripheral blood derived from monocytic leukemia patients).

\section{DCs}

DCs are a very important immune cell type as they present antigens (i.e., antigen-presenting cells [APCs]) to the $\mathrm{T}$ cells. For this reason, they have been described as the 'bridge' between the innate (antigen uptake) and the adaptive (antigen presentation) immune system. Their main function is to take up antigens, to process them (by proteolytic cleavage) and to present the fragments to the $T$ cells via receptor-mediated mechanisms; the latter occurs via the MHC II receptor, a receptor widely present on the surface of human DCs, in combination with co-stimulatory molecules and cytokines [65].

Regarding in vitro experiments, isolation of monocytes from human buffy coat is common practice, as well as their differentiation into mature DCs (monocyte derived dendritic cells: MDDCs), with the addition of growth factors such as granulocyte-monocyte colony stimulating factor and IL-4 [66].

T cells

$T$ cells play a key role in adaptive immunity, since they communicate with DCs; activated DCs present the antigen fragment on the MHC-II receptor which possesses high affinity for the T-cell receptor. T cells can be subdivided into two major types: helper $\mathrm{T}$ cells $\left(\mathrm{CD}^{+}\right.$; modulation of the immune responses) and cytotoxic $\mathrm{T}$ cells $\left(\mathrm{CD}^{+}\right.$; destroying bacterial infected, cancer and apoptotic cells) $[67,68]$. Upon interacting with DCs, $\mathrm{T}$ cells are activated and they differentiate toward different fates, depending on the signal obtained from the DCs (antigenic specificity) [69].

For in vitro studies, $T$ cells can be generated from human buffy coat from the CD14-negative fraction $\left(\mathrm{CD} 14^{-}\right)$and purified, with the $\mathrm{CD} 4$ positive fraction $\left(\mathrm{CD}^{+}\right)$being used for generation of human $\mathrm{T}$ cells in vitro [70]. The most common approach for measuring T-cell activation and proliferation in vitro is via radiolabeled compounds (such as thymidine, which is integrated into the DNA upon replication of the cells), as described by Blank et al. in 2011 [70].

$B$ cells

Briefly, B cells represent the second class of lymphocytes involved in the adaptive immune system and are mainly associated with antibody-based responses [67]. Mature naive B cells mainly circulate through lymphoid organs in the periphery until they are activated 
by an encounter with their cognate antigen, inducing production and secretion of antibodies specific for individual antigens.

Similar to $\mathrm{T}$ cells, isolation of $\mathrm{B}$ cells can be performed via magnetic separation from human buffy coat in vitro (characterized by expression of surface markers CD19/CD20). The lineage of human B cells and their role in immunity in the respiratory system has recently been reviewed and described in detail [71].

\section{NP interactions with lung immune cells in vitro}

Generally, the interaction of NPs with immune cells is determined by the physicochemical properties of the NPs, predominantly by their surface coatings (reviewed recently $[28,31])$. At present, there is a lack of data on the immune responses to nanosized particles coming into contact with the respiratory system. Not only cell viability, cytotoxicity and cytokine secretion but also phenotypic and activation marker expression, as well as antigen uptake and processing capacities, need to be examined and considered in order to fully understand the effect of NPs on immune cells. Undoubtedly, data on their immune-stimulatory or immune-suppressive effects will determine the fate of novel therapeutic and diagnostic NP-based approaches $[19,24,29,31,70]$.

\section{Monoculture cell studies Macrophages}

In general, macrophages readily take up NPs, therefore they are frequently the cells of interest in various diagnostic and therapeutic studies, for example, in the metal NP-based imaging and targeting of cells associated with various human diseases [24,72-74]. To date, various micro- or nano-sized systems have been devised as promising drug vehicles targeting pulmonary macrophages via specific surface receptor recognition-based direction or passively (based on the high phagocytosis activity of macrophages) [45,75]. Regarding specific macrophage targeting, the most extensively studied are the various biodegradable polymer-based NPs. It has been suggested that inflammatory responses depend on uptake (internalization) pathways, as demonstrated on an example of PLGA NPs, which elicit lower inflammatory cytokine production in murine macrophages J774 compared with microparticles of the same composition [76].

In addition, other types of NPs, such as lipid-latexbased NPs, have been proposed as promising platforms for specific imaging of proinflammatory macrophages in various lung disease diagnostics [75]. Also, singlewalled carbon nanotubes were reported to be biodegradable by the action of the THP-1 human monocytic cell line [77].

\section{DCs}

DCs are frequently applied as novel nanovaccine approaches in experimental immunotherapy studies for the treatment of cancer and infectious diseases [78,79]. Similar to macrophages, polymer-based nanocarriers, such PLGA NPs, have been proposed as suitable for the targeting of DCs and subsequent activation of T cells [41-43]. The outcomes of our previous study suggested that MDDCs exposed to poly(vinyl alcohol)coated SPIONs caused downregulation of antigen processing and $\mathrm{CD}^{+}$T-cell stimulation, thus reverting the DCs to a more functionally immature state [70]. It has been reported that NP shape, size and surface charge have a significant impact on DCs; in particular, a higher phagocytic activity has been reported for positively charged particles. With respect to this, our group has recently demonstrated that NP surface modifications can significantly modulate uptake by human monocyte-derived DCs, however, it is difficult to relate this to cellular responses [80].

\section{B \& T lymphocytes}

There are few studies on the interaction of NPs with $\mathrm{T}$ and $\mathrm{B}$ cells, and an even smaller number of studies reporting NP-influenced antibody production by $\mathrm{B}$ cells. There have been some reports on the activation of naive B cells by antigen-loaded calcium-phosphatebased NPs [81], as well as on the impact of engineered NPs on cell activation and subsequent induction of antibody production by B cells (reviewed in [31]). Gold NPs were found to induce activation of mouse spleenderived B cells and promote immunoglobulin G secretion in vitro, ex vivo and in vivo [82], whereas a single exposure to iron oxide NPs attenuated antigen-specific antibody production [83]. It has been shown that gold NPs can influence T-cell proliferation both in vitro and in vivo [82]. On the other hand, a recent study showed that gold NPs did not affect T-cell function and proliferation, suggesting gold NP labeled T cells can be used in NP-coupled immunotherapy, for example, for trafficking lung-resident cancer cells [84]. However, any further impact of NPs on B-lymphocyte function, such as the induction and/or inhibition of antibody production, remains unclear [31,72]. It has also been reported that gold NPs themselves do not cause T-cell proliferation, but simultaneous exposure to gold NPs and antigens can have a synergistic effect in terms of T-cell proliferation [85]. Moreover, polymer-based nanocarrier systems were shown to deliver antigens to DCs, resulting in an increased cytokine production and thus enhanced $\mathrm{CD}^{+} \mathrm{T}$-cell priming, which suggests that oxidation-sensitive polymersomes can function as a vaccine delivery platform for inducing cell-mediated antigen-specific immune responses [86]. 
3D lung cell model studies

Since inhalation is widely accepted as the primary route of entry for aerosolized NPs into the human body [4], accurate and realistic studies need to be performed in order to examine the effects of inhaled NPs on lung cells [87-89]. Advanced in vitro co-culture models are becoming increasingly promising, since they provide a more ethical, cost-effective and faster alternative to in vivo models.

Overall, 3D co-culture models exposed to air-liquid interface conditions are very well characterized models that provide accurate data, by virtue of them being very similar to the in vivo setting (Figure 3 ; $[90]$ ). Complementary to this, $3 \mathrm{D}$ co-cultures can be aerosolized with NP suspensions, after which numerous endpoints such as cellular uptake, functional and immunological properties can be measured [88,89,91,92]. Apart from the need for good cellular models, sophisticated instruments that can deliver NP aerosols in a dose-controlled manner are also mandatory, for instance the air-liquid interface cell exposure system or the commercially available Vitrocell ${ }^{\circledR}$ Cloud.

\section{In vitro \& in vivo settings}

In some recent in vitro monoculture studies on DCs, the absence of an immunological effect was observed with gold and iron oxide NPs $[70,80,93]$ at concentrations up to $20 \mu \mathrm{g} / \mathrm{ml}$, whereas there is evidence that certain NP types at higher concentrations can result in alterations of the antigen uptake and processing capacities, which could result in T-cell proliferation [53,70,85]. However, it is difficult to correlate cellular uptake with biological endpoints because different NP types can elicit different biological responses [56,80].

Both in vitro and in vivo studies report that the size and surface of an NP play the most important roles in its immunomodulatory effect [94]. For example, 20-nm polystyrene NPs were found to enhance T-cell proliferation in lung-draining lymph nodes of mice, while 1000 $\mathrm{nm}$ polystyrene NPs failed to induce T-cell proliferation [25]. In addition, $7 \mathrm{~nm}$ gold NPs were detected in the blood of the experimental mice at higher concentrations than when $20 \mathrm{~nm}$ gold NPs were used [61].

From an in vivo and organ translocation/biodistribution perspective, Kreyling and colleagues observed

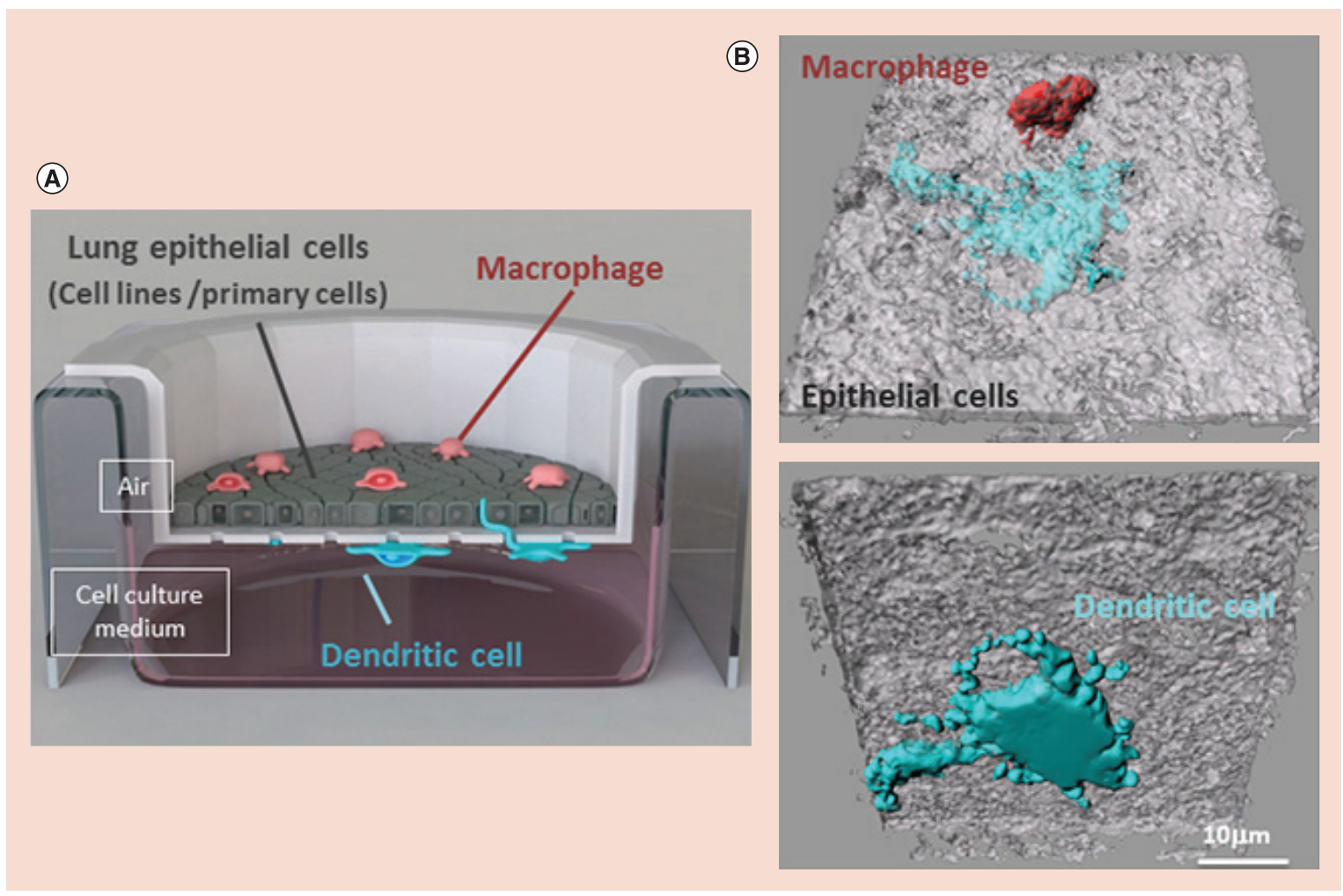

Figure 3. (A) Schematic drawing of the triple-cell co-culture model composed of epithelial cells, monocyte-derived macrophages and dendritic cells. The cells are exposed to air in the upper chamber and to cell culture medium in the lower part. (B) Laser scanning images of the model. Epithelial cells (white, volume rendering), macrophages (red, surface rendering), and dendritic cells (light blue, surface rendering) are shown. The same data set is shown from above (upper image) and from below (lower image). Adapted with permission from [90]. 
higher amounts of positively charged gold NPs in the lungs after $24 \mathrm{~h}$ of intratracheal instillation when compared with the use of their negatively charged counterparts [16,61]. The surface charge of gold NPs can influence translocation in vivo, with $-\mathrm{COO}^{-}$-functionalized gold NPs having a significantly higher translocation rate than the $-\mathrm{NH}_{3}{ }^{+}$-functionalized gold NPs. This effectively means that the blood circulation is higher for the $-\mathrm{COO}^{-}$-functionalized particles, whereas the $-\mathrm{NH}_{3}{ }^{+}$ functionalized particles are retained in the organs [16]. Similarly, alveolar macrophages and different subpopulations of pulmonary DCs preferentially captured positively charged gold NPs compared with their negatively charged counterparts in mice in vivo, and also induced enhanced OVA-specific $\mathrm{CD}^{+}{ }^{+}$T-cell stimulation [95]. However, the dose reaching the targeted cells also needs to be considered; for instance, SPIONs, administered via inhalation pathways, were found to induce dose-related expression of some similar inflammatory markers in murine macrophages both in vitro and in vivo, whereas discrepancies were observed for nonimmunological, respiratory epithelial cells [96]. Nevertheless, the aspect of the biocorona on NPs has to be deciphered in the assessment of NP delivery to the respiratory system [97].

It is important to mention that immunizing animals with specific NPs, even in the presence of strong adjuvants, is frequently reported to be unsuccessful in terms of generating NP-specific antibodies, however, it can be successfully induced by conjugation of NPs to a protein carrier (discussed in [31]).

Nevertheless, it should be noted that NP-based vaccines combined with adjuvants can elicit a strong cellular immune response upon viral infections, for example, activation of T-cell priming. However, cancer-based vaccines also aim to activate $\mathrm{CD} 8^{+} \mathrm{T}$ cells. In this respect, biodegradable NPs are considered an attractive delivery system since they can deliver high yields of peptides and drugs along with adjuvants to the site of interest, and thus achieve an effective immune response [98].

\section{Future perspective}

Currently, extensive research is being devoted to the design of novel nanotechnology-based approaches for the inhalation-based treatment of a range of pulmonary diseases, particularly for targeting lung-resident immune cells. Although many drugs are already available on the market to treat pulmonary disorders and various novel nanocarrier types have been proposed, more investigation is needed: especially regarding the specific targeting of single immune cell types in human lungs. Indeed, biomedicine can significantly benefit from the use of nanotechnology-based carriers, yet proper risk assessment of such novel materials will require thorough characterization of their immunomodulatory effects, either desired or unintentional.

Most of the present studies are performed using in vitro approaches (using either human or murine derived cells), or in vivo studies on rodents. Due to the general desire to reduce animal experiments, and the ethical questions regarding human in vivo studies, the application of sophisticated in vitro approaches using relevant immune and lung epithelial cells will present an increasingly important step in the evaluation of biomedical NPs.

From an immunotoxicology perspective, it is of critical importance to understand if an organism recognizes NPs as foreign material (antigen), thereby triggering innate immune responses [97]. Despite the scientific advances made over the last decade in the area of NP-immune system interactions, the development of nanocarrier vaccines that can result in strong cellular immune responses will be key for the improvement of clinical outcomes.

\section{Financial \& competing interests disclosure}

This work was supported by the Swiss National Science Foundation NRP64 program on 'Opportunities and Risks of Nanomaterials', by the Swiss National Science Foundation through the National Centre of Competence in Research Bio-Inspired Materials, and the Adolphe Merkle Foundation. The authors have no other relevant affiliations or financial involvement with any organization or entity with a financial interest in or financial conflict with the subject matter or materials discussed in the manuscript apart from those disclosed.

No writing assistance was utilized in the production of this manuscript.

\section{Executive summary}

- Combination of the anatomical features of the human respiratory tract with the unique physicochemical properties of nanoparticles (NPs) can serve as an adequate basis for the development of novel inhalative NPbased approaches for the treatment of various lung diseases.

- NP-based drug delivery is a rapidly emerging research field requiring a highly interdisciplinary approach by groups of experts encompassing material scientists, physicists, biologists as well as clinicians, in order to meet the requirements for (bio)medical applications.

- Well-defined and characterized in vitro model systems of single immune cells - but also more complex 3D lung models involving immune cells - represent suitable approaches for the assessment of NP-immune system interactions and thus contribute to the development of the 'nanoimmuno'-based therapeutic approaches. 
Open access

This work is licensed under the Creative Commons Attribution

\section{References}

Papers of special note have been highlighted as: $\bullet$ of interest;

•• of considerable interest

1 Zhao L, Seth A, Wibowo N et al. Nanoparticle vaccines. Vaccine 32(3), 327-337 (2014).

2 Baetke SC, Lammers T, Kiessling F. Applications of nanoparticles for diagnosis and therapy of cancer. Brit. J. Radiol. 88, 1054 (2015).

3 Fenniri $\mathrm{H}$. The Canadian regenerative medicine and nanomedicine enterprise (CARMENE). Int. J. Nanomed. 1(3), 225-227 (2006).

4 Oberdorster G, Stone V, Donaldson K. Toxicology of nanoparticles: a historical perspective. Nanotoxicology 1(1), 2-25 (2007).

5 Mills NL, Donaldson K, Hadoke PW et al. Adverse cardiovascular effects of air pollution. Nat. Clin. Pract. Card. 6(1), 36-44 (2009).

6 Oberdorster G. Pulmonary effects of inhaled ultrafine particles. Int. Arch. Occup. Environ. Health 74(1), 1-8 (2001).

7 Müller L, Lehmann AD, Johnston BD et al. Inhalation pathway as a promising portal of entry: what has to be considered in designing new nanomaterials for biomedical application? In: Handbook of Nanotoxicology, Nanomedicine and Stem Cell Use in Toxicology. Sahu SC, Casciano DA (Eds). John Wiley \& Sons, Ltd, Chichester, UK (2014).

8 Gehr P, Bachofen M, Weibel ER. Normal human lung ultrastructure and morphometric estimation of diffusion capacity. Resp. Physiol. 32(2), 121-140 (1978).

9 Weibel ER. Morphometry of the human-lung - state of the art after 2 decades. B. Eur. Physiopath. Res. 15(5), 999-1013 (1979).

10 Heyder J, Gebhart J, Rudolf G, Schiller CF, Stahlhofen W. Deposition of particles in the human respiratory-tract in the size range 0.005-15-Mu-M. J. Aerosol. Sci. 17(5), 811-825 (1986).

11 Patton JS, Byron PR. Inhaling medicines: delivering drugs to the body through the lungs. Nat. Rev. Drug. Discov. 6(1), 67-74 (2007).

- Provides an in-depth discussion of the advantages of the physiological features of the lung for medical applications, such as systemic delivery of therapeutics via inhalation.

12 Schurch S, Gehr P, Hof VI, Geiser M, Green F. Surfactant displaces particles toward the epithelium in airways and alveoli. Resp. Physiol. 80(1), 17-32 (1990).

13 Gehr P, Schürch S, Berthiaume Y, Hof VI, Geiser M. Particle retention in airways by surfactant. J. Aerosol. Med. Pulm. Drug. Deliv. 3(1), 27-43 (2009).

14 Semmler-Behnke M, Takenaka S, Fertsch S et al. Efficient elimination of inhaled nanoparticles from the alveolar region: evidence for interstitial uptake and subsequent reentrainment onto airways epithelium. Environ. Health. Perspect. 115(5), 728-733 (2007).
4.0 License. To view a copy of this license, visit http://creativecommons.org/licenses/by/4.0/

15 Nemmar A, Hoet PHM, Vanquickenborne B et al. Passage of inhaled particles into the blood circulation in humans. Circulation 105(4), 411-414 (2002).

16 Kreyling WG, Hirn S, Moller W et al. Air-blood barrier translocation of tracheally instilled gold nanoparticles inversely depends on particle size. ACS Nano 8(1), 222-233 (2014).

- An in vivo study that reports that the translocation of gold nanoparticles (NPs) across the air-blood barrier, intratracheally instilled into rats, depends on the NP surface charge and specific surface area.

17 Oberdorster G, Sharp Z, Atudorei V et al. Translocation of inhaled ultrafine particles to the brain. Inhal. Toxicol. 16(6-7), 437-445 (2004).

18 Alexis F, Pridgen E, Molnar LK, Farokhzad OC. Factors affecting the clearance and biodistribution of polymeric nanoparticles. Mol. Pharmaceut. 5(4), 505-515 (2008).

19 Hardy CL, Lemasurier JS, Belz GT et al. Inert 50-nm polystyrene nanoparticles that modify pulmonary dendritic cell function and inhibit allergic airway inflammation. J. Immunol. 188(3), 1431-1441 (2012).

20 Kopf M, Schneider C, Nobs SP. The development and function of lung-resident macrophages and dendritic cells. Nat. Immunol. 16(1), 36-44 (2015).

21 Holt PG, Strickland DH, Wikstrom ME, Jahnsen FL. Regulation of immunological homeostasis in the respiratory tract. Nat. Rev. Immunol. 8(2), 142-152 (2008).

22 Vermaelen K, Pauwels R. Pulmonary dendritic cells. Am. J. Resp. Crit. Care. 172(5), 530-551 (2005).

23 Upham JW, Stick SM, Moodley D. Lung cell biology. In: Pediatric Respiratory Medicine (2nd Edition). Taussig LM, Landau LI (Eds). Mosby Inc., an affiliate of Elsevier Inc., PA, USA, 35-44 (2008).

24 Azarmi S, Roa WH, Loebenberg R. Targeted delivery of nanoparticles for the treatment of lung diseases. Adv. Drug Deliver. Rev. 60 (8), 863-875 (2008).

- Provides an overview of the late 20th century beginnings of the uses of NPs for pulmonary drug delivery systems, and thus establishes the basis for the current advanced drug delivery systems in human lungs.

25 Blank F, Stumbles PA, Seydoux E et al. Size-dependent uptake of particles by pulmonary antigen-presenting cell populations and trafficking to regional lymph nodes. Am. J. Resp. Cell. Mol. 49(1), 67-77 (2013).

26 Smith DM, Simon JK, Baker JR. Applications of nanotechnology for immunology. Nat. Rev. Immunol. 13(8), 592-605 (2013).

27 Park YM, Lee SJ, Kim YS et al. Nanoparticle-based vaccine delivery for cancer immunotherapy. Immune Netw. 13(5), 177-183 (2013).

28 Kononenko V, Narat M, Drobne D. Nanoparticle interaction with the immune system. Arh. Hig. Rada. Toksiko. 66(2), 97-108 (2015). 

Safety and Effects (1st Edition). Academic Press, an imprint of Elsevier, Oxford, UK, 138 (2013). understanding of interactions between nanoparticles and the immune system. Toxicol. Appl. Pharmacol. 299, 78-89 (2016).

31 Ilinskaya AN, Dobrovolskaia MA. Understanding the immunogenicity and antigenicity of nanomaterials: Past, present and future. Toxicol. Appl. Pharmacol. 299, 70-77 (2016).

-. A review of current knowledge regarding the immunogenicity and antigenicity of engineered NPs. Provides a concise description of the response of immune cells to NPs, and research directions for nanotechnologyand biotechnology-based therapeutics. nanoparticles with immunocompetent cells: nanosafety considerations. Nanomedicine (Lond.) 7(1), 121-131 (2012).

34 Mahon E, Salvati A, Bombelli FB, Lynch I, Dawson KA. Designing the nanoparticle-biomolecule interface for 'targeting and therapeutic delivery'. J. Control. Release 161(2), 164-174 (2012).

35 Moore TL, Rodriguez-Lorenzo L, Hirsch V et al. Nanoparticle colloidal stability in cell culture media and impact on cellular interactions. Chem. Soc. Rev. 44(17), 6287-6305 (2015)

36 Hirsch V, Kinnear C, Moniatte M, Rothen-Rutishauser B, Clift MJD, Fink A. Surface charge of polymer coated SPIONs influences the serum protein adsorption, colloidal stability and subsequent cell interaction in vitro. Nanoscale 5(9), 3723-3732 (2013).

37 Tian FR, Clift MJD, Casey A et al. Investigating the role of shape on the biological impact of gold nanoparticles in vitro. Nanomedicine (Lond.) 10(17), 2643-2657 (2015).

38 Dreaden EC, Alkilany AM, Huang XH, Murphy CJ, El-Sayed MA. The golden age: gold nanoparticles for biomedicine. Chem. Soc. Rev. 41(7), 2740-2779 (2012).

39 Li JL, Wang L, Liu XY et al. In vitro cancer cell imaging and therapy using transferrin-conjugated gold nanoparticles. Cancer Lett. 274(2), 319-326 (2009).

40 Papasani MR, Wang GK, Hill RA. Gold nanoparticles: the importance of physiological principles to devise strategies for targeted drug delivery. Nanomedicine 8(6), 804-814 (2012).

41 Keijzer C, Spiering R, Silva AL et al. PLGA nanoparticles enhance the expression of retinaldehyde dehydrogenase enzymes in dendritic cells and induce FoxP3(+) T-cells in vitro. J. Control. Release 168(1), 35-40 (2013).

42 Saluja SS, Hanlon DJ, Sharp FA et al. Targeting human dendritic cells via DEC-205 using PLGA nanoparticles leads to enhanced cross-presentation of a melanoma-associated antigen. Int. J. Nanomed. 9, 5231-5246 (2014).

43 Rosalia RA, Cruz LJ, Van Duikeren S et al. CD40-targeted dendritic cell delivery of PLGA-nanoparticle vaccines induce potent anti-tumor responses. Biomaterials 40, 88-97 (2015).
44 Cimalla P, Werner T, Winkler K et al. Imaging of nanoparticle-labeled stem cells using magnetomotive optical coherence tomography, laser speckle reflectometry, and light microscopy. J. Biomed. Opt. 20 (3), 036018 (2015).

45 Lee WH, Loo CY, Traini D, Young PM. Nano- and microbased inhaled drug delivery systems for targeting alveolar macrophages. Expert Opin. Drug Del. 12(6), 1009-1026 (2015).

- The authors conclude that in order to facilitate drug delivery, there is still a need for an in-depth identification of more target-specific receptors as well as a consideration of the toxicity of nanocarriers arising from prolonged residence in the lung.

46 Seydoux E, Fytianos K, Von Garnier C, Rothen-Rutishauser B, Blank F. Targeting immune cells (Chapter 8d). In: The ISAM Textbook of Aerosol Medicine (1st Edition). Dhand R, Rothen-Rutishauser B, Hickey AJ (Eds). International Society of Aerosols in Medicine (ISAM)/Mary Ann Libert Publishing, Inc., NY, USA (2016).

47 Csaba N, Garcia-Fuentes M, Alonso MJ. Nanoparticles for nasal vaccination. Adv. Drug Deliver. Rev. 61(2), 140-157 (2009).

48 Ryan GM, Kaminskas LM, Kelly BD, Owen DJ, Mcintosh MP, Porter CJH. Pulmonary administration of PEGylated polylysine dendrimers: absorption from the lung versus retention within the lung is highly size-dependent. $\mathrm{Mol}$. Pharmaceut. 10(8), 2986-2995 (2013).

49 Muttil P, Pulliam B, Garcia-Contreras L et al. Pulmonary immunization of guinea pigs with diphtheria CRM-197 antigen as nanoparticle aggregate dry powders enhance local and systemic immune responses. AAPS J. 12(4), 699-707 (2010).

50 Thomas C, Rawat A, Hope-Weeks L, Ahsan F. Aerosolized PLA and PLGA nanoparticles enhance humoral, mucosal and cytokine responses to hepatitis B vaccine. Mol. Pharmaceut. 8(2), 405-415 (2011).

51 Taratula O, Garbuzenko OB, Chen AM, Minko T. Innovative strategy for treatment of lung cancer: targeted nanotechnology-based inhalation co-delivery of anticancer drugs and siRNA. J. Drug Target. 19(10), 900-914 (2011).

52 Carrillo-Conde B, Song EH, Chavez-Santoscoy A et al. Mannose-functionalized 'pathogen-like' polyanhydride nanoparticles target C-type lectin receptors on dendritic cells. Mol. Pharmaceut. 8(5), 1877-1886 (2011).

53 Stano A, Nembrini C, Swartz MA, Hubbell JA, Simeoni E. Nanoparticle size influences the magnitude and quality of mucosal immune responses after intranasal immunization. Vaccine 30(52), 7541-7546 (2012).

54 Chiodo F, Marradi M, Park J et al. Galactofuranosecoated gold nanoparticles elicit a pro-inflammatory response in human monocyte-derived dendritic cells and are recognized by DC-SIGN. ACS Chem. Biol. 9(2), 383-389 (2014).

55 Seydoux E, Rothen-Rutishauser B, Nita IM et al. Sizedependent accumulation of particles in lysosomes modulates dendritic cell function through impaired antigen degradation. Int. J. Nanomed. 9, 3885-3902 (2014). 
56 Rodriguez-Lorenzo L, Fytianos K, Blank F, Von Garnier C, Rothen-Rutishauser B, Petri-Fink A. Fluorescence-encoded gold nanoparticles: library design and modulation of cellular uptake into dendritic cells. Small 10(7), 1341-1350 (2014).

57 Dekali S, Gameza C, Kortulewskic T, Blazya K, Ratb P, Lacroixa G. Assessment of an in vitro model of pulmonary barrier to study the translocation of nanoparticles. Toxicol. Rep. 1, 157-171 (2014).

58 Chaplin DD. Overview of the immune response. J. Allergy. Clin. Immun. 125(2), S3-S23 (2010).

59 Turvey SE, Broide DH. Innate immunity. J. Allergy Clin. Immun. 125(2), S24-S32 (2010).

60 Bonilla FA, Oettgen HC. Adaptive immunity. J. Allergy Clin. Immun. 125(2), S33-S40 (2010).

61 Balasubramanian SK, Poh KW, Ong CN, Kreyling WG, Ong WY, Yu LE. The effect of primary particle size on biodistribution of inhaled gold nano-agglomerates. Biomaterials 34(22), 5439-5452 (2013).

62 Balhara J, Gounni AS. The alveolar macrophages in asthma: a double-edged sword. Mucosal. Immunol. 5(6), 605-609 (2012).

63 Mosser DM, Edwards JP. Exploring the full spectrum of macrophage activation. Nat. Rev. Immunol. 8(12), 958-969 (2008).

64 Hamilton JA. Colony-stimulating factors in inflammation and autoimmunity. Nat. Rev. Immunol. 8(7), 533-544 (2008).

65 Banchereau J, Briere F, Caux C et al. Immunobiology of dendritic cells. Annu. Rev. Immunol. 18, 767-811 (2000).

66 Lehner M, Morhart P, Stilper A, Holter W. Functional characterization of monocyte-derived dendritic cells generated under serumfree culture conditions. Immunol. Lett. 99(2), 209-216 (2005).

67 Janeway CaJ, Travers P, Walport M, Shlomchik MJ. Immunobiology. The Immune System in Health and Disease (5th Edition). Garland Science, NY, USA (2001).

68 Bosco N, Kirberg J, Ceredig R, Agenes F. Peripheral T cells in the thymus: have they just lost their way or do they do something? Immunol. Cell. Biol. 87(1), 50-57 (2009).

69 Nakayamada S, Takahashi H, Kanno Y, O'shea JJ. Helper T cell diversity and plasticity. Curr. Opin. Immunol. 24(3), 297-302 (2012).

70 Blank F, Gerber P, Rothen-Rutishauser B et al. Biomedical nanoparticles modulate specific $\mathrm{CD} 4\left(^{+}\right) \mathrm{T}$ cell stimulation by inhibition of antigen processing in dendritic cells. Nanotoxicology 5(4), 606-621 (2011).

71 Kato A, Hulse KE, Tan BK, Schleimer RP. B-lymphocyte lineage cells and the respiratory system. J. Allergy Clin. Immun. 131(4), 933-957 (2013).

72 Luo YH, Chang LW, Lin PP. Metal-based nanoparticles and the immune system: activation, inflammation, and potential applications. Biomed. Res. Int. 143720, 1-12 (2015).

73 Chellat F, Merhi Y, Moreau A, Yahia L. Therapeutic potential of nanoparticulate systems for macrophage targeting. Biomaterials 26(35), 7260-7275 (2005).
74 Hirst SM, Peairs AD, Gogal R, Seal S, Reilly CM. Cerium oxide nanoparticles decrease inflammation in $\mathrm{J} 774$ cells. FASEB J. 22, 758.2 (2008).

75 Bagalkot V, Badgeley MA, Kampfrath T, Deiuliis JA, Rajagopalan S, Maiseyeu A. Hybrid nanoparticles improve targeting to inflammatory macrophages through phagocytic signals. J. Control. Release 217, 243-255 (2015).

76 Nicolete R, Dos Santos DF, Faccioli LH. The uptake of PLGA micro or nanoparticles by macrophages provokes distinct in vitro inflammatory response. Int. Immunopharmacol. 11(10), 1557-1563 (2011).

77 Kagan VE, Kapralov AA, St Croix CM et al. Lung macrophages 'digest' carbon nanotubes. Using a superoxide/ peroxynitrite oxidative pathway. ACS Nano 8(6), 5610-5621 (2014).

78 Cruz LJ, Tacken PJ, Rueda F, Domingo JC, Albericio F, Figdor CG. Targeting nanoparticles to dendritic cells for immunotherapy. Method. Enzymol. 509, 143-163 (2012).

79 Le Guevel X, Palomares F, Torres MJ, Blanca M, Fernandez TD, Mayorga C. Nanoparticle size influences the proliferative responses of lymphocyte subpopulations. RSC Adv. 5(104), 85305-85309 (2015).

80 Fytianos K, Rodriguez-Lorenzo L, Clift MJD et al. Uptake efficiency of surface modified gold nanoparticles does not correlate with functional changes and cytokine secretion in human dendritic cells in vitro. Nanomedicine 11(3), 633-644 (2015).

81 Temchura VV, Kozlova D, Sokolova V, Uberla K, Epple $\mathrm{M}$. Targeting and activation of antigen-specific B-cells by calcium phosphate nanoparticles loaded with protein antigen. Biomaterials 35(23), 6098-6105 (2014).

82 Lee CH, Syu SH, Chen YS et al. Gold nanoparticles regulate the blimp1/pax 5 pathway and enhance antibody secretion in B-cells. Nanotechnology 25(12), 125103 (2014).

83 Shen CC, Wang CC, Liao MH, Jan TR. A single exposure to iron oxide nanoparticles attenuates antigen-specific antibody production and T-cell reactivity in ovalbumin-sensitized BALB/c mice. Int. J. Nanomed. 6, 1229-1235 (2011).

84 Meir R, Shamalov K, Betzer O et al. Nanomedicine for cancer immunotherapy: tracking cancer-specific T-Cells in vivo with gold nanoparticles and CT imaging. ACS Nano 9(6), 6363-6372 (2015).

85 Liptrott NJ, Kendall E, Nieves DJ et al. Partial mitigation of gold nanoparticle interactions with human lymphocytes by surface functionalization with a 'mixed matrix'. Nanomedicine (Lond.). 9(16), 2467-2479 (2014).

86 Scott EA, Stano A, Gillard M, Maio-Liu AC, Swartz MA, Hubbell JA. Dendritic cell activation and T cell priming with adjuvant- and antigen-loaded oxidation-sensitive polymersomes. Biomaterials 33(26), 6211-6219 (2012).

87 Lehmann A, Brandenberger C, Blank F, Gehr P, RothenRutishauser B. A 3D model of the human epithelial airway barrer. In: Methods in Bioengineering. Alternative Technologies to Animal Testing. Maguire T, Novik E (Eds). Artech House Boston, London, UK, 239-260 (2010).

88 Rothen-Rutishauser BM, Kiama SG, Gehr P. A threedimensional cellular model of the human respiratory tract to 
study the interaction with particles. Am. J. Resp. Cell. Mol. 32(4), 281-289 (2005).

-• Description and characterization of the 3D lung in vitro system, as represented by epithelial cells, macrophages and dendritic cells, suitable for investigation of particle interactions with lung immune cells.

Blank F, Rothen-Rutishauser BM, Schurch S, Gehr P. An optimized in vitro model of the respiratory tract wall to study particle cell interactions. J. Aerosol. Med. 19(3), 392-405 (2006).

90 Rothen-Rutishauser B, Lehmann AD, Clift MJD, Blank F, Gehr P. Laser scanning microscopy combined with image restoration to analyse a 3D model of the human epithelial airway barrier. Swiss. Med. Wkly. 140, 3-9 (2010).

91 Lenz AG, Karg E, Lentner B et al. A dose-controlled system for air-liquid interface cell exposure and application to zinc oxide nanoparticles. Part. Fibre. Toxicol. 6, 32 (2009).

92 Giese C, Marx U. Human immunity in vitro - solving immunogenicity and more. Adv. Drug. Deliver. Rev. 69 103-122 (2014).

93 Villiers CL, Freitas H, Couderc R, Villiers MB, Marche PN. Analysis of the toxicity of gold nano particles on the immune system: effect on dendritic cell functions. J. Nanopart. Res. 12(1), 55-60 (2010).

94 Braakhuis HM, Park MVDZ, Gosens I, De Jong WH, Cassee FR. Physicochemical characteristics of nanomaterials that affect pulmonary inflammation. Part. Fibre Toxicol. 11, 18 (2014)

95 Seydoux E, Rodriguez-Lorenzo L, Blom RaM et al. Pulmonary delivery of cationic gold nanoparticles boost antigen-specific $\mathrm{CD}^{+}{ }^{+} \mathrm{T}$ cell proliferation Nanomedicine: NBM 12(7), 1815-1826 (2016)

96 Teeguarden JG, Mikheev VB, Minard KR et al. Comparative iron oxide nanoparticle cellular dosimetry and response in mice by the inhalation and liquid cell culture exposure routes. Part. Fibre Toxicol. 11, 46 (2014).

97 Fadeel B. Clear and present danger? Engineered nanoparticles and the immune system. Swiss. Med. Wkly 142 , w13609 (2012).

98 Silva JM, Videira M, Gaspar R, Preat V, Florindo HF. Immune system targeting by biodegradable nanoparticles for cancer vaccines. J. Control. Release 168(2), 179-199 (2013). 